\title{
GRETA THUNBERG COMO PERSONAJE VIRALIZADOR DE LOS TUIT DEL SECTOR INFORMATIVO DURANTE LA CUMBRE DEL CLIMA COP25
}

\section{GRETA THUNBERG AS A VIRAL CHARACTER IN THE TWEETS OF THE INFORMATION SECTOR DURING THE COP25 CLIMATE SUMMIT}

(8) $\mathrm{R}^{\mathrm{G}}$ Rafael Carrasco Polaino. Universidad Complutense de Madrid. España.

(8) $\mathbb{R}^{R}$ Ernesto Villar Cirujano. Universidad Villanueva. España.

\section{RESUMEN}

El objetivo de la investigación fue determinar en qué medida los medios de información publicaron contenidos relacionados con Greta Thunberg durante la cumbre del clima COP25 y cómo fueron las interacciones de esta activista con los medios a través de la red social Twitter, además de analizar el modo en el que los medios de información se valieron del personaje para aumentar su impacto en la red social. Se clasificaron los tuits publicados por los medios de información en relación con la variable mención a Greta Thunberg, a través de pruebas estadísticas no paramétricas, y se estudió cómo los tuits que mencionaban a la activista sueca generaban más o menos engagement que los tuits que no lo hacían. Además, se analizó si el engagement de los diferentes mensajes publicados por los medios lograban engagement diferentes en función de si estos incluían elementos multimedia, URLs o sólo texto. La conclusión principal es que los tuits que incluían mención al usuario @GretaThunberg generaron mayor engagement que los que sólo incluían su nombre, además de que la activista en ningún momento interactuó con las cuentas que la mencionaron. Por otro lado, los tuits con elementos multimedia y los que nos incluían URLs generaron mayor engagement que el resto.

PALABRAS CLAVE: COP25; Twitter; Greta Thunberg; engagement; periodismo; cambio climático.

\begin{abstract}
The objective of the investigation was to determine to what extent the information media published content related to Greta Thunberg and how this protagonist's interactions with the media were through the social network Twitter, in addition to determining to what extent the information media were used the character to increase their impact on the social network. After classifying the tweets published by the media in relation to the variable mentioning Greta Thunberg, through non-parametric statistical tests, it was studied to what extent the tweets that mentioned Greta Thunberg generated more or less engagement than the tweets that did not. they did. In addition, it was analyzed whether the engagement of the different messages published by the
\end{abstract}

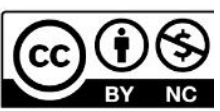

Recibido: 23/11/2020 - Aceptado: 03/03/2021 - Publicado: 05/05/2021 
media achieved different engagement depending on whether they included multimedia elements, URLs, or only text. The main conclusion is that the tweets that included a mention of Greta Thunberg's user generated greater engagement than those that only included her name, in addition to the fact that the activist never interacted with the accounts that mentioned her. On the other hand, tweets with multimedia elements and those that included URLs generated more engagement than the rest.

KEYWORDS: COP25; Twitter; Greta Thunberg; engagement; journalism; climate change

\section{Cómo citar el artículo:}

Carrasco Polaino, R. y Villar Cirujano, E. (2021). El papel de los medios de comunicación en la Greta Thunberg como personaje viralizador de los tuit del sector informativo durante la cumbre del clima COP25. Revista de Ciencias de la Comunicación e Información, 26, 1-13. http://doi.org/10.35742/rcci.2021.26.e116

\section{INTRODUCCIÓN Y OBJETIVOS}

Cada vez que se produce un acontecimiento de alcance global, tanto si es algo inesperado como si se trata de un evento organizado con antelación, las redes sociales se convierten en un activo foro de intercambio de información y de debate, un fenómeno abordado desde múltiples enfoques (Kim, Hastak, 2018; Hassan et al, 2018; Demszky et al, 2019; Guallar et al, 2016) y a través de ejemplos concretos de viralización en eventos y movimientos sociales, ya sea específicamente en cumbres climáticas anteriores (Segerberg, Bennet, 2011) como en cualquier otro tema de debate (Shiau Chou et al, 2020) o acontecimiento global, como la reciente pandemia de la covid-19 (Noor et al, 2020).

Este flujo de información es especialmente llamativo en Twitter, mucho más si el tema es percibido como un asunto de interés general y cuenta con la participación de actores que dinamizan la discusión y aglutinan en torno a ellos la atención. Un ejemplo de ello ha sido la Cumbre Mundial del Clima COP25 celebrada en Madrid (España) en diciembre de 2019, en la que han estado presentes los dos elementos mencionados. Por un lado, el cambio climático, que se ha asentado en el centro de la agenda política y ciudadana, y cuya huella en las redes sociales ya ha sido estudiada en cumbres anteriores (Hopke, Hestres, 2018; Tyagi, Babcock y Carley, 2019). Por el otro, la irrupción en la conversación de la mediática activista sueca Greta Thunberg, presente en la cumbre, cuya relevancia es incuestionable tanto en Twitter como en el debate social y político.

En el primer caso, su cuenta verificada acumula 4,5 millones de seguidores y cerca de 9.000 tuits publicados en dos años y medio (datos a enero de 2021). Además, en las semanas previas a la cumbre esta red social fue uno de sus principales vehículos de comunicación, como por ejemplo durante su travesía en barco, que fue seguida a través de la cuenta @Sailing_LaVaga y de la etiqueta \#LaVagabonde, el nombre del catamarán en el que viajaba. 
En cuanto a su repercusión social y mediática, Thunberg ha sido nominada en dos ocasiones (2019 y 2020) al Premio Nobel de la Paz, fue una de las ganadoras del Premio Right Livelihood 2019 (también conocido como el "Nobel Alternativo") tres meses antes de la COP25 y dos días antes de que terminara la cumbre de Madrid fue desginada persona del año por la revista Time, la más joven en recibir este reconocimiento en los 93 años de historia de este galardón. Además, ha hablado ante los principales mandatarios y con alguno de ellos ha mantenido reuniones oficiales, como la canciller de Alemania, Angela Merkel.

Más allá de las aportaciones individuales de los usuarios de Twitter, una buena parte de la discusión en esta red social se articula en torno a lo que publican los medios de comunicación, que ven cada vez más en esta red social (Nielsen, Schroder, 2014) un interesante canal para aumentar el tráfico de sus informaciones, dentro de su papel, adaptado a los nuevos tiempos, de fijar la agenda pública (Skogerbo, Krumsvik, 2014) seleccionando los temas de debate -gatekeeping- (McQuail, 2016) y adaptando el concepto político de agenda-setting (Alonso-Muñoz; Casero-Ripollés, 2018) a los nuevos formatos y las propuesta de temas que hacen los propios usuarios (Singer, 2014; Coddington, Holton, 2014). Y en cuanto a la vertiente de activismo, sin duda las redes sociales son para las ONG un altavoz de primer orden (Blight, Ruppel, Schoenbauer, 2017; Carrasco-Polaino, Villar-Cirujano, Martín-Cárdaba, 2018).

Con estos cuatro elementos (la red social Twitter, la Cumbre del Clima, los medios y la activista más mediática), la presente investigación pretende dar respuesta a una serie de cuestiones:

1. ¿En qué medida los medios de información publicaron contenidos relacionados con Greta Thunberg?

2. ¿Recurrieron de una forma habitual a mencionarla directamente en sus post con el ánimo de aumentar el engagement?

3. ¿Dio resultado esta estrategia, es decir, la mención a Greta Thunberg se tradujo en un mayor engagement de los tuits?

4. En un plano formal, ¿en qué medida contenían estos mensajes vídeo, fotografía, memes o solo texto?

5. ¿La elección de uno u otro formato influía en la viralización del tuit?

6. ¿Cómo fue la interacción de Greta Thunberg con los medios a través de Twitter, si es que la hubo?

\section{METODOLOGÍA}

Para responder a estas preguntas e hipótesis, se descargaron a través del programa NodeXI pro (Smith, 2010) todos los tuits y sus respectivas interacciones (respuestas, menciones y retuits) que contenían el hashtag \#cop25 entre el 2 de diciembre y el 12 de diciembre de 2019, fechas en las que se celebró la cumbre.

De la base de datos generada con todas las interacciones $(n=67.431)$, se filtraron aquellos mensajes e interacciones publicados por los medios de información y periodistas que tuvieran su cuenta de usuario de Twitter verificada $(n=350)$ y las interacciones de Greta Thunberg con las de los emisores. Con estos datos se generó un grafo para determinar la relación que los diferentes medios mantuvieron con Greta 
Thunberg y a la inversa (menciones, respuestas y retuits) en relación con el tema del evento.

En un segundo paso se calculó el engagement generado por cada una de las acciones. El engagement en Twitter se define, generalmente, como la tasa de interacción. Se trata de un índice que mide la capacidad de un tuit de generar interés o acción en Twitter. Es un índice relevante, porque además de establecer una relación emocional sobre los usuarios tiene valor a la hora de que el algoritmo de Twitter muestre contenido de manera orgánica en los muros de los diferentes usuarios con los que se ha tenido interacción (Tornos Inza, 2020). No se trata del número de seguidores, sino del grado de implicación o de interés que los seguidores presentan antes ciertas publicaciones (Montells, 2016). Dos son las maneras más extendidas para el cálculo del engagement o tasa de interacción: en la primera de ellas, el total de las interacciones recibidas por un tuit es dividido por el número de impresiones (el número de personas que lo han recibido en su muro o timeline). En la segunda, el total de interacciones que recibe el tuit es dividido entre el número de seguidores del autor del tuit (Tornos Inza, 2020; Montells, 2016). De esta forma y por el tipo de datos recogidos y su estructura, se decidió por la segunda fórmula mencionada. Así, la operación utilizada para el cálculo del engagement quedó establecida como engagement $=\left((\right.$ total de interacciones recibidas $) / \mathrm{n}^{\circ}$ de seguidores $){ }^{*} 100$ (HerreraTorres et al., 2017)

Para cada publicación se realizó un análisis de contenido (Piñuel-Raigada, 2002) a través de una ficha previamente diseñada que constaba de los siguientes ítems:

- Tipo de cuenta: para determinar si el usuario emisor era un medio de comunicación corporativo o un periodista profesional desde su cuenta particular.

- Tipo de publicación: se distinguió entre los tuits originales publicados, las menciones, las respuestas, los retuits y las menciones en retuits.

- Contiene elementos multimedia: De esta forma se distinguió entre las publicaciones que tenían insertado en su cuerpo una imagen, un vídeo o un GIF animado y las que no contenían ninguno de estos recursos.

- Contiene URL: Si distinguieron las publicaciones que contenían en su cuerpo una URL que pudieran pulsarse y ésta dirigiera a una página o elemento distinto del tuit y de sus interacciones.

- Menciona a Greta Thunberg: Se identificaron aquellas publicaciones que incluían en su cuerpo el nombre de Greta Thunberg de aquellas que no lo hacían.

- Se menciona a @gretathunberg: En este caso se seleccionaron las publicaciones que en su cuerpo incluyeran directamente el identificador de la cuenta de Greta Thunberg: @gretathunberg.

Para analizar el engagement de cada post en función de las diferentes variables antes se identificaron y filtraron aquellas publicaciones que presentaban un engagement atípico (outliers) a través del cálculo de las puntuaciones típicas del engagement de cada post. Al ser la muestra de más de 80 registros se decidió filtrar aquellos post con una puntuación típica con un valor superior al valor absoluto de 3 (Estrada et al., 2004). 
Se identificaron 6 registros con un engagement atípico que fueron filtrados y no se procesaron junto con el resto de cara al análisis estadístico inferencial.

Para determinar la aplicación del tipo de pruebas estadísticas que permitieran ver cómo variaba el engagement en relación con cada una de las otras variables y si las diferencias presentadas resultaban estadísticamente significativas, se realizaron pruebas de normalidad a través del sistema de Kolmogorov-Smirnov (Massey, 1951) o de Shapiro-Wilk (Royston, 1992) en función de si la muestra de cada grupo dentro de cada una de las variables era mayor o menor de 50 registros.

Las pruebas de normalidad mostraron resultados negativos, por lo que se decidió hacer un análisis no paramétrico a través de la prueba $U$ de Mann-Whitney (MacFarland et al., 2016) en el caso de presentar la variable a analizar dos grupos de Kruskal-Wallis (Ostertagová et al., 2014), cuando las variables presentaban más de dos grupos.

\section{ANÁLISIS DE RESULTADOS}

\subsection{Análisis de frecuencias}

Tras el filtrado de los datos descargados se encontraron 18 periodistas con cuentas verificadas y 122 medios que cumplían la misma condición que habían publicado algún tuit durante la celebración de la COP25 utilizando la etiqueta \#COP25.

El análisis de las frecuencias de estas publicaciones presentaba los siguientes resultados:

Tabla 1. Tabla de frecuencias.

\begin{tabular}{|l|l|l|}
\hline Tipo de cuenta & Frecuencia & Porcentaje \\
\hline $\begin{array}{l}\text { Medios de comunicación y } \\
\text { programas }\end{array}$ & 305 & 87,1 \\
\hline Periodistas & 45 & 12,9 \\
\hline Total & $\mathbf{3 5 0}$ & $\mathbf{1 0 0 , 0}$ \\
\hline Tipo de publicación & Frecuencia & Porcentaje \\
\hline Tuit & 186 & 53,1 \\
\hline Mención & 124 & 35,4 \\
\hline Respuesta & 3 & 0,9 \\
\hline Retuit & 12 & 3,4 \\
\hline Mención en retuit & 25 & 7,1 \\
\hline Total & $\mathbf{3 5 0}$ & $\mathbf{1 0 0 , 0}$ \\
\hline $\begin{array}{l}\text { Contiene elementos } \\
\text { multimedia }\end{array}$ & Frecuencia & Porcentaje \\
\hline Sí & 175 & 50,0 \\
\hline No & 175 & 50,0 \\
\hline Total & $\mathbf{3 5 0}$ & $\mathbf{1 0 0 , 0}$ \\
\hline Contiene URL & Frecuencia & Porcentaje \\
\hline Sí & 221 & 63,1 \\
\hline No & 129 & 36,9 \\
\hline Total & $\mathbf{3 5 0}$ & $\mathbf{1 0 0 , 0}$ \\
\hline Se menciona a Greta Thunberg & Frecuencia & Porcentaje \\
\hline No & 284 & 81,1 \\
\hline & &
\end{tabular}




\begin{tabular}{|l|l|l|}
\hline Sí & 66 & 18,9 \\
\hline Total & $\mathbf{3 5 0}$ & $\mathbf{1 0 0 , 0}$ \\
\hline Se incluye @gretathunberg & Frecuencia & Porcentaje \\
\hline No & 332 & 94,9 \\
\hline Sí & 18 & 5,1 \\
\hline Total & 350 & 100,0 \\
\hline
\end{tabular}

Fuente: elaboración propia.

Los 140 usuarios analizados realizaron diferentes tipos de acciones en Twitter durante la celebración de la Cumbre del Clima. De todas las publicaciones e interacciones realizadas (350), los periodistas individuales realizaron el 12,9\% (45) del total de todo el sector informativo y los medios de información el 87,1\% (305).

El reparto de las 350 interacciones no resultó homogéneo. El 53,1\% de las acciones fueron tuits originales, el 35,4\% (124) menciones a otros usuarios, el 7,1\% (25) retuits en donde se incluía la mención a otros usuarios, 3,4\% (12) retuits y $0,9 \%$ (3) respuestas a otros.

En cuanto a la inserción de un elemento multimedia, ya fuera en formato imagen o en formato vídeo dentro del propio cuerpo de la publicación, los datos arrojaron resultados completamente homogéneos, ya que la mitad de las publicaciones contenían un elemento multimedia y la otra mitad no lo incluían.

La inserción de URL que llevaran a un contenido externo presentó unos resultados en los que el 63,1\% (221) de las publicaciones contenía alguna URL en la que el usuario podía pulsar y salir de la publicación concreta con destino a otra web o publicación. El $36,9 \%$ (129) restante eran publicaciones en donde no se podía pulsar en enlace alguno por no ofrecerse, por lo que sólo permitían al usuario ver las interacciones con esa publicación en concreto.

Respecto a las menciones a Greta Thunberg en Twitter por parte del sector informativo, el 18,9\% (66) del total mencionaba a la activista en el cuerpo de la publicación o mencionaba su usuario de Twitter, enlazando con ello la publicación con la cuenta de usuario.

Cuando se aislaron las publicaciones que mencionaban a Greta Thunberg por su identificador, filtrando las que la mencionan por insertar su nombre en el cuerpo del tuit, los datos mostraron que el 5,1\% (18) del total de las publicaciones tenían esta característica.

Es decir, casi tres de cada cuatro usuarios que aluden a Thunberg lo hacen sin mencionar su perfil. Esta omisión, consciente o inconsciente, puede deberse a diversas motivaciones: que el usuario prefiera comentar "de espaldas" a la activista porque no quiera interactuar con ella, que considere que hacer una mención directa a Thunberg no le reportará ningún beneficio, que no haya tenido en cuenta los posibles efectos positivos de la mención en la viralización, que no quiera dedicar tiempo a buscar su cuenta oficial... Sea cual sea la explicación, resulta imposible determinarla científicamente. 


\subsection{Análisis del engagement}

Tras realizar las diferentes pruebas estadísticas que ayudarán a relacionar el engagement generado por cada tuit, respuesta, mención, retuit o mención en retuit en función de cada una de las variables que se han descrito con anterioridad, los resultados obtenidos se muestran y describen en las siguientes tablas de datos:

Tabla 2. Engagement en función del tipo de publicación.

\begin{tabular}{|l|l|l|l|}
\hline Tipo de publicación & Media & $\mathbf{N}$ & Desviación estándar \\
\hline Tuit & $0,120 \%$ & 181 & $0,359 \%$ \\
\hline Mención & $0,127 \%$ & 122 & $0,378 \%$ \\
\hline Respuesta & $0,215 \%$ & 3 & $0,310 \%$ \\
\hline Retuit & $0,537 \%$ & 12 & $0,917 \%$ \\
\hline Mención en retuit & $0,142 \%$ & 25 & $0,367 \%$ \\
\hline Total & $\mathbf{0 , 1 3 9 \%}$ & $\mathbf{3 4 3}$ & $\mathbf{0 , 4 0 2} \%$ \\
\hline
\end{tabular}

Fuente: elaboración propia.

El retuit es el tipo de interacción que más engagement generó $(m=0,53 \%$; ds $=0,91 \%)$ seguido de las respuestas $(m=0,21 \%$; $d s=0,31 \%)$. Quedan por detrás en cuanto a engagement logrado las menciones en retuit $(m=0,145 ; d e=0,36 \%)$, las menciones $(m=0,127 \% ; d s=0,37 \%)$ y los tuits originales $(m=0,120 \% ; d s=0,35 \%)$. Sin embargo, la prueba de Kruskal-Wallis no indicó que las diferencias en el engagement fueran lo suficientemente amplias como para considerarse significativas $(p=0,52)$.

Tabla 3. Engagement en función de los elementos multimedia.

\begin{tabular}{|l|l|l|l|}
\hline Tiene multimedia & Media & $\mathbf{N}$ & Desviación estándar \\
\hline Sí & $0,142 \%$ & 172 & $0,399 \%$ \\
\hline No & $0,137 \%$ & 171 & $0,406 \%$ \\
\hline Total & $\mathbf{0 , 1 3 9 \%}$ & $\mathbf{3 4 3}$ & $\mathbf{0 , 4 0 2} \%$ \\
\hline
\end{tabular}

Fuente: elaboración propia.

La prueba $U$ de Mann-Whitney tampoco arrojó diferencias significativas a la hora de analizar el engagement como factor modificado por la inserción de elementos multimedia en el cuerpo de las publicaciones $(U=14.729,5 ; p=0,98)$, a pesar de que las publicaciones que sí insertaron algún tipo de elemento multimedia, ya fuera vídeo, imagen o GIF animado, obtuvieron un engagement mayor $(m=0,142 \%$; $d s=0,39 \%)$ que los que no lo hicieron ( $m=0,137 \%$; $d s=0,40 \%)$.

Tabla 4. Engagement en función de las URL.

\begin{tabular}{|l|l|l|l|}
\hline Tiene URL & Media & $\mathbf{N}$ & Desviación estándar \\
\hline Sí & $0,096 \%$ & 217 & $0,361 \%$ \\
\hline No & $0,214 \%$ & 126 & $0,456 \%$ \\
\hline Total & $\mathbf{0 , 1 3 9 \%}$ & $\mathbf{3 4 3}$ & $\mathbf{0 , 4 0 2} \%$ \\
\hline
\end{tabular}

Fuente: elaboración propia. 
El hecho de que las publicaciones incluyeran una URL en su cuerpo que dirigía a una web o contenido ajeno sí que tuvo efectos estadísticamente relevantes en el engagement $(U=17.522,5 ; p<0,001)$. Los post con $U R L$ obtuvieron un engagement menor ( $m=0,096 \%$; $d s=0,36 \%)$ que aquellos que no permitían abandonar la publicación o sus interacciones directas a través de una URL $(m=0,21 \%$; $d s=0,45 \%)$.

Tabla 5. Engagement en función de Greta Thunberg.

\begin{tabular}{|l|l|l|l|}
\hline $\begin{array}{l}\text { Menciona a Greta } \\
\text { Thunberg }\end{array}$ & Media & $\mathbf{N}$ & Desviación estándar \\
\hline No & $0,141 \%$ & 277 & $0,428 \%$ \\
\hline Sí & $0,132 \%$ & 66 & $0,265 \%$ \\
\hline Total & $\mathbf{0 , 1 3 9 \%}$ & $\mathbf{3 4 3}$ & $\mathbf{0 , 4 0 2} \%$ \\
\hline
\end{tabular}

Fuente: elaboración propia.

El hecho de que se mencionara a Greta Thunberg en el contenido de la publicación no tuvo efectos relevantes en el engagement de las diferentes publicaciones $(U=10.101,5 ; p=0,18)$. Las publicaciones que incluyeron el nombre de la joven activista en su contenido obtuvieron un engagement menor $(m=0,13 \% ; d s=0,26 \%)$ que las que no lo hicieron ( $m=0,13 \%$; $d s=0,40 \%)$.

Tabla 6. Engagement en función de @gretathunberg.

\begin{tabular}{|l|l|l|l|}
\hline $\begin{array}{l}\text { Menciona a } \\
\text { @gretathunberg }\end{array}$ & Media & $\mathbf{N}$ & Desviación estándar \\
\hline No & $0,133 \%$ & 325 & $0,403 \%$ \\
\hline Sí & $0,257 \%$ & 18 & $0,371 \%$ \\
\hline Total & $\mathbf{0 , 1 3 9 \%}$ & $\mathbf{3 4 3}$ & $\mathbf{0 , 4 0 2} \%$ \\
\hline
\end{tabular}

Fuente: elaboración propia.

Sin embargo, sí que obtuvieron un engagement mayor las publicaciones que incluyeron el identificador @gretathunberg en su contenido, ya redactaran el nombre de Greta Thunberg o no. Las primeras obtuvieron un engagement medio de 0,25\% con desviación estándar de $0,37 \%$, mientras que las que no apuntaban directamente a la cuenta de usuario de la activista obtuvieron un engagement menor $(m=0,13 \%$, $\mathrm{ds}=0,40 \%$ ). Estas diferencias se mostraron como estadísticamente significativas $(U=3.742 ; p<0,05)$.

\section{DISCUSIÓN Y CONCLUSIONES}

Los tuits originales generaron menos engagement que cualquier otro tipo de interacción, aunque las diferencias no fueron significativas. A pesar de estas diferencias no relevantes, sí que se podría haber respondido a las publicaciones de los usuarios además de hacer retuit, buscando un engagement mayor en las cuentas de medios de comunicación y de periodistas. Publicar contenidos originales en Twitter y no hacer un seguimiento de ellos interaccionando con los usuarios y respondiendo a sus observaciones y comentarios no permite exprimir el potencial de la red social. 
A la hora de preparar una publicación en Twitter, no fue relevante en cuanto al engagement si en ella se incluyó algún elemento multimedia, ya fuera imagen, vídeo o GIF animado, ya que esta característica no tuvo efectos en el engagement logrado. Sin embargo, sí que la inserción de una URL en el contenido tuvo efectos negativos. Al insertar una URL se está ofreciendo al receptor una alternativa para abandonar la conversación o la red social y mandarle a una web externa. Como estrategia informativa podría ser correcta si estos enlaces se dirigen a una información 0 contenido del propio medio de comunicación o del periodista, porque a pesar de reducirse la interacción con el tuit se aumentan las visitas en la web de destino a la que dirige la URL, cumpliendo así con el objetivo fundamental por el que los medios recurren a esta red de microblogging.

En relación con este aspecto, si el plan de los medios y los periodistas era utilizar Twitter como herramienta de promoción de sus contenidos propios alojados en sus respectivas webs, la estrategia de insertar enlaces a sus informaciones se podría considerar correcta. Sin embargo, si el objetivo era utilizar Twitter como canal de información y conversación autónomo, la inserción de una URL en las publicaciones podría haber resultado un error.

Los temas tratados por el sector informativo en Twitter durante la celebración de la cumbre de clima fueron variados y no se centraron exclusivamente en el personaje de Greta Thunberg (se la menciona en el 19\% del total de las publicaciones).

Greta Thunberg no reaccionó a ninguna de las menciones recibidas, ni respondió, hizo retuit o mencionó en retuit, lo que redujo de forma drástica el engagement potencial de las publicaciones en Twitter de las cuentas del sector informativo. Seguramente, si alguno de los post publicados por medios o periodistas que hubieran mencionado la cuenta @gretathunberg hubieran tenido alguna reacción por parte de ella, estos tuits se hubieran viralizado mucho más. Se debe recordar que cuando un usuario es mencionado en cualquier tuit le llega un aviso, mientras que si en lugar del identificador de la cuenta del usuario lo que se incluye es su nombre en el cuerpo de la publicación, este aviso no se ejecuta, por lo que es difícil que la persona mencionada realice alguna acción sobre esta publicación (no sabe que ha sido mencionada), reduciendo con ello su viralización potencial. Por este motivo podría considerarse un error por parte del sector informativo no haber incluido en las publicaciones que trataban temas relacionados con Greta Thunberg su cuenta de usuario en lugar de su nombre como texto. No obstante, resulta imposible determinar en qué casos la ausencia de mención directa es un acto voluntario o involuntario, aunque la lógica lleve a pensar en esto último en lo referente a los medios de comunicación.

La acción de los periodistas y medios de comunicación en Twitter durante la cumbre climática celebrada en 2019 en Madrid se centró en la difusión de contenidos propios a través de URLs insertados en sus tuits, sin destinar esfuerzos posteriores a mantener la conversación sobre los temas publicados. La conversación no se concentró en el personaje de Greta Thunberg, pero cuando sí se comunicó sobre ella no se aprovechó el potencial de contar con la participación de un personaje tan mediático en el evento, al no incluirse su nombre de usuario en los tuits. 
Pese a las limitaciones obvias que todo análisis de un evento concreto lleva implícitas, como podría ser el reducido marco temporal o las limitaciones de las herramientas para la obtención de datos completos que superen las barreras impuestas por las redes sociales, las conclusiones de este estudio muestran la conveniencia que para los medios de comunicación puede tener centrar sus esfuerzos en Twitter en decisiones sencillas que no requieren de una profunda formación en redes sociales, como no incluir URLs externas para no desviar el tráfico o mencionar directamente la cuenta de un personaje mediático relacionado con el tema de discusión para así aumentar el engagement, en lugar de hacer una mención indirecta. Posteriores estudios sobre otros temas de discusión en los que coincidan los dos elementos ahora analizados (un acontecimiento global y un personaje de gran repercusión mediática) mostrarán si estos patrones se repiten, se matizan o se modifican.

\section{REFERENCIAS}

Alonso-Muñoz, L., \& Casero-Ripollés, A. (2018). Communication of European populist leaders on twitter: Agenda setting and the "more is less" effect. Profesional de la Información, 27(6), 1193-1202. https://doi.org/10.3145/epi.2018.nov.03

Blight, M. G., Ruppel, E. K., \& Schoenbauer, K. V. (2017). Sense of Community on Twitter and Instagram: Exploring the Roles of Motives and Parasocial Relationships. Cyberpsychology, Behavior, and Social Networking, 20(5), 314-319. https://doi.org/10.1089/cyber.2016.0505

Carrasco-Polaino, R., Villar-Cirujano, E., \& Martín-Cárdaba, M. Á. (2018). Artivismo y ONG: Relación entre imagen y «engagement» en Instagram. Comunicar, 26(57). https://doi.org/10.3916/C57-2018-03

Coddington, M., \& Holton, A. E. (2014). When the Gates Swing Open: Examining Network Gatekeeping in a Social Media Setting. Mass Communication and Society, 17(2), 236-257. https://doi.org/10.1080/15205436.2013.779717

Demszky, D., Garg, N., Voigt, R., Zou, J., Gentzkow, M., Shapiro, J., \& Jurafsky, D. (2019). Analyzing Polarization in Social Media: Method and Application to Tweets on 21 Mass Shootings. NAACL HLT 2019 - 2019 Conference of the North American Chapter of the Association for Computational Linguistics: Human Language Technologies - Proceedings of the Conference, 1, 2970-3005. https://doi.org/10.3145/epi.2016.may.05

Estrada, A., Batanero, C., \& Fortuny, J. M. (2004). Un estudio sobre conocimientos de estadística elemental de profesores en formación. Educación Matemática, 16(1), 89-111.

Guallar, J., Suau, J., Ruiz-Caballero, C., Sáez, A., \& Masip, P. (2016). Redistribución de noticias y debate público en las redes sociales. El profesional de la información, 25(3), 358-366. https://doi.org/10.3145/epi.2016.may.05

Hassan, N. Y., Gomaa, W. H., Khoriba, G. A., \& Haggag, M. H. (2018). Supervised Learning Approach for Twitter Credibility Detection. Proceedings - 2018 13th 
International Conference on Computer Engineering and Systems, (ICCES), 196201. https://doi.org/10.1109/ICCES.2018.8639315

Herrera-Torres, J. C., Pérez-Tur, F., García-Fernández, J. \& Fernández-Gavira, J. (2017). El uso de las redes sociales y el engagement de los clubes de la Liga Endesa ACB. Cuadernos de psicología del deporte, 17 (3), 175-182. https://revistas.um.es/cpd/article/view/313981

Hopke, J. E., \& Hestres, L. E. (2018). Visualizing the Paris Climate Talks on Twitter: Media and Climate Stakeholder Visual Social Media During COP21. Social Media + Society, 4(3), 205630511878268. https://doi.org/10.1177/2056305118782687

Kim, J., \& Hastak, M. (2018). Social network analysis: Characteristics of online social networks after a disaster. International Journal of Information Management, 38(1), 86-96. https://doi.org/10.1016/.i.jinfomgt.2017.08.003

MacFarland, T. W., \& Yates, J. M. (2016). Mann-Whitney U Test. En T. W. MacFarland \& J. M. Yates, Introduction to Nonparametric Statistics for the Biological Sciences Using R (pp. 103-132). Springer International Publishing. https://doi.org/10.1007/978-3-319-30634-6 4

Massey, F. J. (1951). The Kolmogorov-Smirnov Test for Goodness of Fit. Journal of the American Statistical Association, 46(253), 68-78. https://doi.org/10.1080/01621459.1951.10500769

McQuail, D. (2016). Mass Communication. In The International Encyclopedia of Political Communication (pp. 1-12). Wiley. https://doi.org/10.1002/9781118541555.wbiepc155

Montells, L. (2016). Engagement en Twitter: qué es y para qué sirve. Metricool. https://bit.ly/2JW459A

Nielsen, R. K., \& Schrøder, K. C. (2014). The Relative Importance of Social Media for Accessing, Finding, and Engaging with News. Digital Journalism, 2(4), 472-489. https://doi.org/10.1080/21670811.2013.872420

Noor, S., Guo, Y., Shah, S. H. H., Fournier-Viger, P., \& Nawaz, M. S. (2020). Analysis of public reactions to the novel Coronavirus (COVID-19) outbreak on Twitter. Kybernetes, ahead-of-print(ahead-of-print). https://doi.org/10.1108/K-05$\underline{2020-0258}$

Ostertagová, E., Ostertag, O., \& Kováč, J. (2014). Methodology and application of the Kruskal-Wallis test. Applied Mechanics and Materials, 611, 115-120. https://doi.org/10.4028/www.scientific.net/AMM.611.115

Piñuel-Raigada, J. L. (2002). Epistemología, metodología y técnicas del análisis de contenido. Estudios de sociolingüística, 3(1), 1-42. https://cutt.ly/PfsYoDAf 
Royston, P. (1992). Approximating the Shapiro-Wilk W-test for non-normality. Statistics and Computing, 2(3), 117-119. https://doi.org/10.1007/BF01891203

Segerberg, A., \& Bennett, W. L. (2011) Social Media and the Organization of Collective Action: Using Twitter to Explore the Ecologies of Two Climate Change Protests. The Communication Review, 14(3), 197-215, https://doi.org/10.1080/10714421.2011.597250

Chou Jen, S., Masanao, O., Takeshi, S., Ken, N., Kanji, S., Junichiro, M., \& Ichiro, S. (2020). Constructive Approach for Early Extraction of Viral Spreading Social Issues from Twitter. 12th ACM Conference on Web Science, 96105. https://doi.org/10.1145/3394231.3397899

Singer, J. B. (2014). User-generated visibility: Secondary gatekeeping in a shared media space. New Media \& Society, 16(1), 55-73. https://doi.org/10.1177/1461444813477833

Skogerbø, E., \& Krumsvik, A. H. (2015). Newspapers, Facebook and Twitter. Journalism Practice, 9(3), 350-366. https://doi.org/10.1080/17512786.2014.950471

Smith, M., Ceni A., Milic-Frayling, N., Shneiderman, B., Mendes Rodrigues, E., Leskovec, J., \& Dunne, C. (2010). NodeXL: a free and open network overview, discovery and exploration add-in for Excel 2007/2010/2013/2016, from the Social Media Research Foundation. https://goo.gl/m5xRJL

Tornos Inza, E. (2020). Tasa de interacción (engagement) en Twitter. Related: Marketing. https://bit.ly/2MIC46r

Tyagi, A., Babcock, M., \& Carley, K. M. (n.d.). Climate Change Debate on Twitter During COP24.

\section{AUTORES}

\section{Rafael Carrasco Polaino}

Profesor Contratado Doctor en el departamento de Periodismo y Nuevos Medios de la Universidad Complutense de Madrid. Imparte asignaturas relacionadas con las Tecnologías de la información, y, en concreto, con los medios digitales y sus herramientas. Cuenta con formación y experiencia en el ámbito del diseño y la comunicación a nivel tanto profesional como académico. En el ámbito de la investigación, su trabajo se centra mayoritariamente en el estudio del social media a través del análisis de redes sociales (ARS) y de la estadística. Estas metodologías de estudio las ha aplicado en investigaciones científicas publicadas en diferentes revistas de impacto. Su labor de investigación centrada en las metodologías antes mencionadas las desarrolla tras su estancia investigadora en el Media Innovation Lab de la Universidad de Viena.

Orcid ID: https://orcid.org/0000-0002-0687-6842

Google Scholar: https://scholar.google.com/citations?user=VxjO4dMAAAAJ\&hl=es

ResearchGate: https://www.researchgate.net/profile/Rafael Carrasco Polaino 
Scopus:

56041715700

https://www.scopus.com/freelookup/form/author.uri?st1=Carrasco\%20Polaino\&st2=R afael

Academia.edu: https://ucm.academia.edu/RafaelCarrasco

\section{Ernesto Villar Cirujano}

Profesor Contratado Doctor en la Universidad Villanueva, de la que es director del Grado en Periodismo. Doctor en Periodismo (UCM) y Máster en Historia Contemporánea por la Universidad Autónoma de Madrid. Profesor de asignaturas de Periodismo Especializado, tecnología y redacción. Miembro de varios proyectos de invetigación y autor de publicaciones científicas en revistas de impacto sobre Periodismo, redes sociales e Historia. Autor de los libros Los espías de Suárez (Espasa, 2015) y Todos quieren matar a Carrero (Libros Libres, 2011). Periodista en activo.

Orcid ID: https://orcid.org/0000-0001-6263-5093

Google Scholar: https://scholar.google.es/citations?user=mt4dn2cAAAAJ\&hl=es

Research Gate: https://www.researchgate.net/profile/Ernesto Cirujano

Academia.edu: https://independent.academia.edu/ErnestoVillarCirujano 